\title{
Análisis clínico y electrofisiológico del hipotiroidismo subclínico en una población hospitalaria
}

\author{
Clinical and electrophysiologic analysis of subclinical \\ hypothyroidism in a hospital population
}

\author{
Proel Pérez-Galdós ${ }^{1,2}$, Rosario Paucar-Cadillo ${ }^{l}$
}

\section{Resumen}

Objetivo: Investigar los efectos del hipotiroidismo primario subclínico y clínico en la función nerviosa periférica. Métodos: Estudio prospectivo de pacientes seleccionados con diagnóstico de hipotiroidismo primario subclínico y clínico con sintomatología sensitiva o motora; se analizaron variables demográficas, tipo de hipotiroidismo, tratamiento con levotiroxina (LT4); se definió la presencia de polineuropatía o neuropatía focal con estudios de velocidad de conducción nerviosa. Resultados: Se estudiaron a 33 pacientes con diagnóstico de hipotiroidismo clínico (HC) y subclínico (HSC), el promedio de edad fue de 64.76 años, el $90.9 \%$ de los pacientes fueron del sexo femenino, el dolor y las parestesias de las extremidades fueron la sintomatologías más frecuentes, en el HC tratados los niveles de TSH y T4 Libre fueron normales, en el HSC los niveles promedio de TSH y T4 libre fueron de: $11.40 \mathrm{mU} / \mathrm{L}$ y $0.85 \mathrm{ng} / \mathrm{dl}$ respectivamente. Al realizar las pruebas neurofisiológicas se observó que el $42.4 \%$ de los pacientes en general, tuvieron alteraciones en los estudios de neuro conducción sensitiva o motora. Conclusiones: Existe un número limitado de estudios funcionales a nivel del Sistema Nervioso Periférico en pacientes con este trastorno, la afectación del nervio periférico en el hipotiroidismo probablemente esté relacionado a la duración de la enfermedad e influenciados por el tratamiento específico.

\section{Palabras clave: Hipotiroidismo, subclínico, electrodiagnóstico, neuropatía, levotiroxina.}

\section{Abstract}

Objective: To investigate the effects of subclinical and clinical primary hypothyroidism on peripheral nerve function. Methods: Prospective study of selected patients diagnosed with subclinical and clinical primary hypothyroidism with sensory or motor symptoms; there were analyzed demographic variables, types of hypothyroidism, treatment with levothyroxine (LT4). The presence of polyneuropathy or focal neuropathy was defined with studies of nerve conduction velocity. Results: We studied 33 patients diagnosed with clinical hypothyroidism $(\mathrm{CH})$ and subclinical $(\mathrm{SCH})$; the average age was 64.76 years; $90.9 \%$ of the patients were female. Pain and paresthesia of the extremities were the most frequent symptomatology. In the CH the levels of TSH and free T4 were normal, in the SCH the average levels of TSH and free T4 were in $11.40 \mathrm{mU} / \mathrm{L}$ and $0.85 \mathrm{ng} / \mathrm{dl}$ respectively. When performing the neurophysiological tests, it was observed that $42.4 \%$ of the patients had alterations in the studies of sensory or motor nerve conduction, in general. Conclusions: There is a limited number of functional studies at the level of the Peripheral Nervous System in patients with this disorder. The peripheral nerve involvement in hypothyroidism is probably related to the duration of the disease and influenced by the specific treatment.

\section{Introducción}

El hipotiroidismo es una enfermedad crónica multisistémica de presentación gradual con síntomas inespecíficos. La causa más frecuente es la disminución en la síntesis y secreción de las hormonas tiroideas y, ocasionalmente, resistencia periférica a éstas ${ }^{(1)}$. El hipotiroidismo es una de las causas más importantes de consulta en endocrinología; afecta frecuentemente al sexo femenino, llegando a comprometer al $2 \%$ de las mujeres adultas. El compromiso masculino es menos frecuente (0.1 al 0.2\%). Según la Encuesta Nacional de Salud y Nutrición de Estados Unidos, la prevalencia de hipotiroidismo es del $0.3 \%$, y de hipotiroidismo subclínico $4.3 \%^{(2)}$. El hipotiroidismo primario ocurre después de la destrucción de la glándula tiroides debido a autoinmunidad, como la tiroiditis de Hashimoto o a 
intervenciones médicas como la cirugía de tiroides, administración de yodo radiactivo o radiación en las zonas del mundo donde el aporte dietético de yodo es suficiente, con un pico entre la cuarta y sexta décadas de la vida ${ }^{(3)}$.

El hipotiroidismo subclínico (HSC) se considera como un trastorno que ocurre en individuos generalmente asintomáticos, y se caracteriza por el hallazgo de cifras elevadas de TSH con niveles normales de hormonas tiroideas. La prevalencia mundial de hipotiroidismo subclínico varía de 1 a 10\%. Las tasas más altas específicas por edad y sexo están en mujeres mayores de 60 años, acercándose al $20 \%$ en algunos informes ${ }^{(4,5)}$. En una encuesta reciente, la prevalencia de hipotiroidismo subclínico en hombres mayores de 74 años fue del $16 \%$, casi tan alta como la de las mujeres de la misma edad, que fue del $21 \%{ }^{(5)}$. Según la intensidad en el incremento de la concentración de TSH, se distinguen 3 grados de HSC: o Grado I: 4,5 - 9,9 mU/L. o Grado II: 10-20 mU/L. o Grado III: >20 $\mathrm{mU} / \mathrm{L}^{(6)}$.

En algunos pacientes con hipotiroidismo clínico (HC), la disfunción nerviosa periférica puede ser la manifestación principal de presentación de la citada patología. Obviamente, la frecuencia y la gravedad de la enfermedad neuromuscular en el hipotiroidismo manifiesto dependen de la gravedad y la duración de la deficiencia de la hormona tiroidea; a nivel del sistema nervioso periférico los síntomas son inespecíficos, pero existen reportes de trastornos en la conducción nerviosa y en la función muscular en pacientes con $\operatorname{HSC}^{(7,8)}$. Los estudios electrodiagnósticos han demostrado una baja conducción y amplitud en los nervios periféricos en pacientes con hipotiroidismo subclínico; a pesar de ello, solo un número limitado de estudios evaluaron las alteraciones funcionales a nivel del sistema nervioso central y periférico en pacientes con este trastorno, con resultados controversiales ${ }^{(9,10)}$. La terapia temprana con levotiroxina (LT4) puede ser útil no solo para proporcionar un tratamiento específico para dichos cambios metabólicos, sino también para evitar la progresión a un hipotiroidismo clínico ${ }^{(11-13)}$. Sin embargo, no se conoce el verdadero rol de los síntomas sensitivos y motores en el hipotiroidismo clínico y subclínico como potenciales predictores de la polineuropatía o neuropatías focales, cuyo tratamiento oportuno sería beneficioso para el paciente.

El propósito del presente estudio se basa en investigar los efectos del hipotiroidismo primario clínico y subclínico en la función nerviosa periférica.

\section{Material y métodos}

\section{Pacientes}

Se seleccionaron prospectivamente pacientes del Servicio de Neurología del Hospital Miguel Grau - EsSalud, que presentaran sintomatología sensitiva o motora con estudios de hormonas tiroideas (TSH y T4 libre) que puedan sugerir un hipotiroidismo subclínico o control post - tratamiento de un hipotiroidismo clínico, durante los años 2017 y 2018 ; el estudio neurofisiológico se llevó a cabo en la Unidad de Neurofisiología del mencionado hospital, registrándose en dichos pacientes las características clínicas, demográficas, tipo de hipotiroidismo y tratamiento con Lt4 .
Se definió la presencia de polineuropatía clínica (PNP) o neuropatía focal (NF), previa evaluación clínica, confirmándose con estudios de neuroconducción sensitiva o motora (NCS). Se excluyó a los enfermos con datos de PNP de cualquier origen o que tuvieran factores de riesgo de PNP como: déficit de vitamina B12, consumo mayor de $40 \mathrm{~g} /$ día de alcohol, diabetes mellitus, factores hereditarios, toma de fármacos neurotóxicos, disfunción hepática, renal o infecciones acompañantes como HIV o TBC y enfermedades malignas. Las evaluaciones clínicas pusieron especial énfasis en los síntomas y signos neurológicos para PNP (parestesias, disestesias, debilidad y/o atrofia muscular, reflejos osteotendinosos, sensibilidad vibratoria y dolorosa), basados en el Neuropathy Symptom Profile (NSP) ${ }^{(14)}$.

El estudio electromiográfico consistió en estudios de conducción nerviosa sensitiva y motora, realizado en un equipo de electromiografía digital de marca Nihon Kohden - modelo Neuropack. Los registros se realizaron con electrodos superficiales. La conducción antidrómica motora y sensitiva se llevó a cabo con estímulos y registro en las zonas habituales. Las frecuencias de estimulación utilizadas fueron de $1 \mathrm{~Hz}$, con una duración de estímulo de $200 \mu$ s y una frecuencia de filtros entre $10 \mathrm{~Hz}$ y $10 \mathrm{kHz}$. El protocolo utilizado fue el diseñado para las polineuropatías, empleado por la mayoría de los autores. Se recogieron las velocidades de conducción sensitiva en metros/segundo y amplitudes en microvoltios de los nervios mediano y cubital. Se obvió el registro del nervio sural en razón a la edad avanzada de la mayoría de los pacientes, la cual podría dar resultados falsos positivos. En la conducción nerviosa motora, se midió la latencia en milisegundos, la velocidad de conducción en metros/segundo y la amplitud en milivoltios de los nervios peroneo, tibial posterior, mediano y cubital. Todas estas mediciones se realizaron con los cuidados de limpieza y temperatura cutáneos recomendados por diversos autores, con el objeto de no aumentar la impedancia y no alterar las velocidades de conducción y/o las amplitudes.

Los criterios electromiográficos de anormalidad en la PNP son la disminución de la amplitud del potencial sensitivo o motor y la disminución de la velocidad de conducción.

\section{Estudio estadístico}

Las variables cuantitativas fueron expresadas en medias y desviación estándar y las variables cualitativas expresadas en porcentajes. Para calcular las diferencias entre pacientes con o sin neuropatía se usaron pruebas no paramétricas con un nivel de significación de $\mathrm{p}:</=0,05$. Para la comparación de las pruebas neurofisiológicas por tipo de hipotiroidismo se seleccionaron aquellas que poseían una distribución normal, mediante la prueba de KolmogorovSmirnov, continuándose con la aplicación de la prueba de ANOVA. El paquete estadístico utilizado para los cálculos fue el SPSS v. 10.0 .

\section{Resultados}

Se estudiaron 33 pacientes con diagnóstico de hipotiroidismo clínico y subclínico; el promedio de edad de ambos grupos fue de 64.76 años (+/- 11.83). El 90.9\% de los pacientes fueron del sexo femenino; el promedio de edad de los 
varones fue de 74.3 años y en las mujeres de 66.3 años. En los pacientes con $\mathrm{HC}$, el promedio de edad fue de $62.37(+/-11.65)$ y en los pacientes con HSC de 68 años (+/-11.69).

La población en su conjunto se dividió en 3 grupos etáreos; la mayor frecuencia de pacientes con HC e HSC se encontró en el rango de $60-79$ años, con el $68.4 \%$ y $57.1 \%$ respectivamente, siendo el HSC más prevalente en el grupo de mayores de 80 años (Tabla 1).

El dolor y las parestesias de las extremidades fueron la sintomatología más frecuente en los dos tipos de hipotiroidismo (Tabla 2).

Se evaluaron los niveles de TSH y T4 libre en la totalidad de los pacientes estudiados, siendo el promedio de TSH sérico de: $5.84 \mathrm{mU} / \mathrm{L}$ (+/- 10.08) (VN: 0.27 - $4.2 \mathrm{mU} / \mathrm{L})$ y el promedio de T4 libre de: $0.97 \mathrm{ng} / \mathrm{dl}(+/-0.19)(\mathrm{VN}: 0.93-$
$1.71 \mathrm{ng} / \mathrm{dl}$ ); en los pacientes con $\mathrm{HC}$ tratados los niveles séricos de TSH y T4 Libre fueron normales y en los pacientes con HSC los niveles promedio de TSH y T4 libre fueron de: $11.40 \mathrm{mU} / \mathrm{L}$ $(+/-14.06)$ y $0.85 \mathrm{ng} / \mathrm{dl}(+/-0.12)$, respectivamente.

Con relación a los grados de intensidad del hipotiroidismo (determinado por los niveles de TSH), el Grado I fue más frecuente en los pacientes con HSC con el 71.4\%, seguidos de los grados II y III con el $14.3 \%$ en cada uno (Tabla 3). La asociación es significativa al comparar los grados de normalidad y anormalidad de los niveles de TSH entre los dos tipos de hipotiroidismo $(\mathrm{p}=0.00)$.

Al realizar las pruebas neurofisiológicas en los dos grupos de pacientes con hipotiroidismo, se observó que el 42.4 $\%$ tuvieron alteraciones en los estudios neurofisiológicos; los pacientes con $\mathrm{HC}$ presentaron un diagnóstico neurofisiológico normal en el $73.7 \%$; el $21.1 \%$ de los casos presentó un sindrome

\begin{tabular}{|c|c|c|c|c|c|c|c|c|}
\hline \multicolumn{9}{|c|}{ Tipo de hipotiroidismo por grupos etáreos } \\
\hline \multirow{2}{*}{ Tipo Hipotiroidismo } & \multicolumn{2}{|c|}{$39-59$ años } & \multicolumn{2}{|c|}{$60-79$ años } & \multicolumn{2}{|c|}{$>80$ años } & \multicolumn{2}{|c|}{ TOTAL } \\
\hline & $\mathbf{N}$ & $\%$ & $\mathbf{N}$ & $\%$ & $\mathbf{N}$ & $\%$ & $\mathbf{N}$ & $\%$ \\
\hline Hipotiroidismo Clínico & 6 & $31.6 \%$ & 13 & $68.4 \%$ & 0 & $0 \%$ & 19 & $100.0 \%$ \\
\hline Hipotiroidismo Subclínico & 3 & $21.4 \%$ & 8 & $57.1 \%$ & 3 & $21.5 \%$ & 14 & $100.0 \%$ \\
\hline TOTAL & 9 & $27.3 \%$ & 21 & $63.6 \%$ & 3 & $9.1 \%$ & 33 & $100.0 \%$ \\
\hline
\end{tabular}

\begin{tabular}{|c|c|c|c|c|c|c|c|c|c|c|}
\hline \multicolumn{11}{|c|}{ Tipo de hipotiroidismo según cuadro clínico } \\
\hline \multirow[t]{2}{*}{ Tipo } & \multicolumn{2}{|c|}{ Asintomático } & \multicolumn{2}{|c|}{$\begin{array}{c}\text { Dolor y } \\
\text { parestesias }\end{array}$} & \multicolumn{2}{|c|}{ Déficit motor } & \multicolumn{2}{|c|}{$\begin{array}{l}\text { Parestesias + } \\
\text { déficit motor }\end{array}$} & \multicolumn{2}{|c|}{ TOTAL } \\
\hline & $\mathbf{N}$ & $\%$ & $\mathbf{N}$ & $\%$ & $\mathbf{N}$ & $\%$ & $\mathbf{N}$ & $\%$ & $\mathbf{N}$ & $\%$ \\
\hline Hipotiroidismo Clínico & 4 & $21.1 \%$ & 14 & $73.7 \%$ & 0 & $0.0 \%$ & 1 & $5.3 \%$ & 19 & $100.0 \%$ \\
\hline Hipotiroidismo Subclínico & 0 & $0.0 \%$ & 11 & $78.6 \%$ & 1 & $7.1 \%$ & 2 & $14.3 \%$ & 14 & $100.0 \%$ \\
\hline TOTAL & 4 & $12.1 \%$ & 25 & $75.8 \%$ & 1 & $3.0 \%$ & 3 & $9.1 \%$ & 33 & $100.0 \%$ \\
\hline
\end{tabular}

\begin{tabular}{|c|c|c|c|c|c|c|c|c|c|c|}
\hline \multicolumn{11}{|c|}{ Tabla 3} \\
\hline \multicolumn{11}{|c|}{ Asociación de los tipo de hipotiroidismo según niveles de TSH } \\
\hline \multirow[t]{2}{*}{ Tipo } & \multicolumn{2}{|c|}{ Normal } & \multicolumn{2}{|c|}{ GRADO I } & \multicolumn{2}{|c|}{ GRADO II } & \multicolumn{2}{|c|}{ GRADO III } & \multicolumn{2}{|c|}{ TOTAL } \\
\hline & $\mathbf{N}$ & $\%$ & $\mathbf{N}$ & $\%$ & $\mathbf{N}$ & $\%$ & $\mathbf{N}$ & $\%$ & $\mathbf{N}$ & $\%$ \\
\hline Hipotiroidismo Clínico & 19 & $100.0 \%$ & 0 & $0.00 \%$ & 0 & $0.0 \%$ & 0 & $0.0 \%$ & 19 & $100.0 \%$ \\
\hline Hipotiroidismo Subclínico & 0 & $0.0 \%$ & 10 & $71.4 \%$ & 2 & $14.3 \%$ & 2 & $14.3 \%$ & 14 & $100.0 \%$ \\
\hline TOTAL & 19 & $57.5 \%$ & 10 & $30.3 \%$ & 2 & $6.1 \%$ & 2 & $6.1 \%$ & 33 & $100.0 \%$ \\
\hline
\end{tabular}

Leyenda: • Grado I: 4,5 - 9,9 mU/L. • Grado II: 10-20 mU/L. • Grado III: >20 mU/L. 


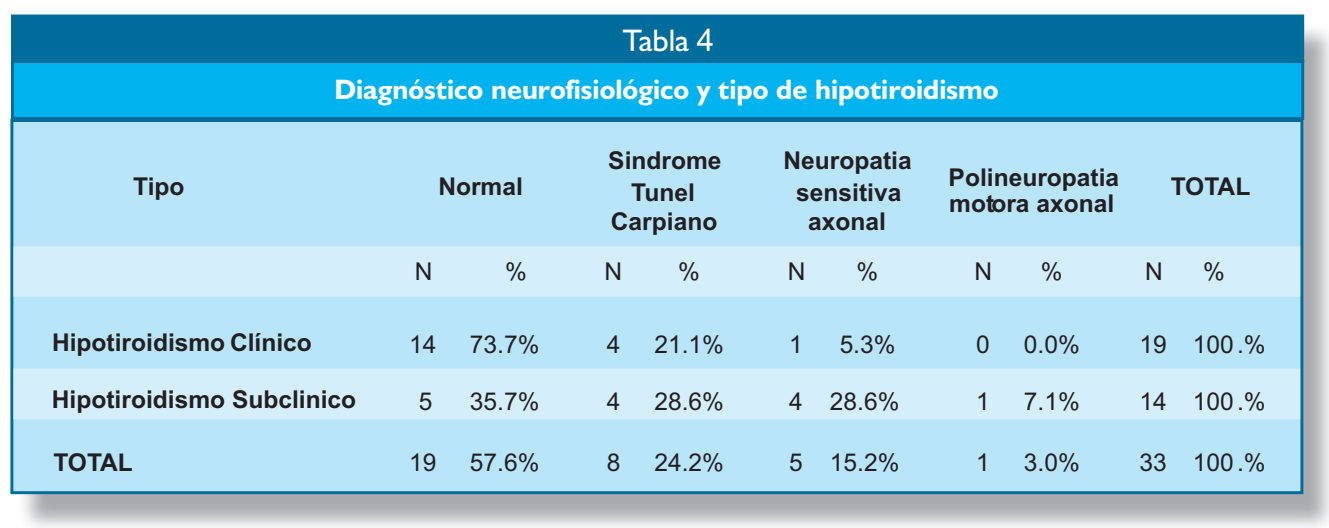

del túnel carpiano, mientras que los pacientes con HSC se distribuyeron casi homogéneamente, con diagnóstico normal en el $35.7 \%$, sindrome del túnel carpiano y neuropatía sensitiva axonal con el $28.6 \%$ de los casos respectivamente. (Tabla 4). La asociación es significativa al comparar los grados de normalidad y anormalidad neurofisiológica con los tipos de hipotiroidismo, siendo predominantemente normales en el HC y con algún diagnóstico neurofisiológico en el HSC $(\mathrm{p}=0.029)$. Por tanto, el riesgo de presentar algún tipo de anormalidad neurofisiológica de los pacientes con HSC frente a los pacientes con $\mathrm{HC}$ es de 5 veces $(\mathrm{OR}=5)$.

El $61.5 \%$ de los pacientes que recibieron tratamiento con LT4 tuvieron pruebas neurofisiológicas normales, en comparación al $42.9 \%$ de los pacientes que no recibieron tratamiento con LT4 y con pruebas neurofisiológicas normales.

Para realizar el análisis comparativo de las pruebas neurofisiológicas aplicadas a los pacientes con HC e HSC, se realizó previamente la prueba de Kolmogorov-Smirnov, para poder aplicar la prueba de ANOVA (Tabla 5). De lo anterior se concluye que la velocidad de conducción motora (VCNM) del nervio cubital es la única prueba que se considera como un grupo homogéneo, compacto y muy diferenciado entre el $\mathrm{HC}$ e HSC por la lejanía de sus medias y su poca variabilidad. Por consiguiente, en la comparación de las medias, mediante el ANOVA se obtiene una diferencia estadísticamente significativa entre las medias de la VCNM cubital del HP (64.5 $\mathrm{m} / \mathrm{s})$ e $\operatorname{HSC}(55.7 \mathrm{~m} / \mathrm{s}) .(\mathrm{p}=0.00)$.

\section{Discusión}

El promedio de edad de los pacientes del estudio fue de 64.76 años, siendo el promedio de edad en el HSC superior al promedio de edad del $\mathrm{HC}$; el sexo femenino fue ampliamente predominante en el estudio en los dos tipos de hipotiroidismo, compatibles con los estudios poblacionales publicados por Hallowell JG et al. ${ }^{(2)}$ y Tunbridge et al. ${ }^{(4)}$ y de acuerdo a las referencias de la literatura universal en estos casos.

Los síntomas predominantes de los pacientes fueron los sensitivos y motores, siendo más evidentes en el HSC en comparación al $\mathrm{HC}$, debido probablemente a que los pacientes con $\mathrm{HC}$ se encontraban recibiendo levo - tiroxina en forma crónica, lo que implicaría una mejora en los intercambios metabólicos existentes entre la musculatura y el nervio periférico de los pacientes hipotiroideos clínicos, en comparación al HSC (los que no recibían tratamiento con levo tiroxina o solo por poco tiempo) y a pesar de no observarse cambios neurofisiológicos significativos entre los dos tipos de hipotiroidismo con el tratamiento en el presente estudio. Se puede inferir entonces que la introducción de una terapia temprana con levo - tiroxina en el HSC podría ser beneficioso para el paciente y evitaría así la progresión hacia un hipotiroidismo clínico ${ }^{(11-13)}$.

En general, el $42.4 \%$ de los pacientes presentaron alteraciones en los estudios de neuroconducción, lo cual es ligeramente inferior a lo reportado por Nebuchennykh $\mathrm{M}^{(5)}$. La polineuropatía sensitiva fue más prevalente en el HSC, similar a lo reportado por otros autores ${ }^{(16-18)}$; Ajeena ${ }^{(19)}$ detectó una alta prevalencia de neuropatía sensorial (44\%), y Balaraman et al. ${ }^{(20)}$ encontraron una reducción significativa en la conducción del nervio sensorial (latencia, amplitud, duración, área, velocidad de conducción) en los nervios mediano y sural, y solo un $10 \%$ de neuropatía puramente motora; en el presente estudio la afectación nerviosa sensitivo motora de tipo axonal fue más prevalente, similar a lo descrito por Duyff et al. ${ }^{(21)}, \mathrm{y}$ contrario a lo descrito por Asia y Warkar ${ }^{(17)}$, en el que predominaba la polineuropatía desmielinizante sensorial.

Las hormonas tiroideas tienen efectos profundos sobre la actividad oxidativa mitocondrial, la síntesis y degradación de proteínas y la sensibilidad a las catecolaminas. En el desarrollo neuronal interactúan una variedad de factores de crecimiento importantes, pudiéndose modular el crecimiento axonal mediante la regulación del ensamblaje de los microtúbulos. Esto puede sugerir un posible mecanismo para la degeneración axonal en el hipotiroidismo.

La prevalencia del síndrome del túnel carpiano (STC) en el presente estudio en general fue del $24.2 \%$, inferior a lo reportado por Karne y Bhalerao ${ }^{(18)}$ y Cruz et al. ${ }^{(22)}$ encontraron que el $60 \%$ y $71,42 \%$ de sus pacientes tenían STC respectivamente. Por otro lado, Ajeena ${ }^{(19)}$ y Asia - Warkar ${ }^{(17)}$ no establecieron el mismo porcentaje alto; esto podría deberse a la diferente metodología neurofisiológica utilizada.

Existe un número limitado de estudios funcionales a nivel del sistema nervioso periférico en pacientes con este trastorno, cuyos resultados son controversiales ${ }^{(9,10)}$. En el presente estudio, al comparar los valores de las pruebas neurofisiológicas en los pacientes con HC y HSC con la prueba 


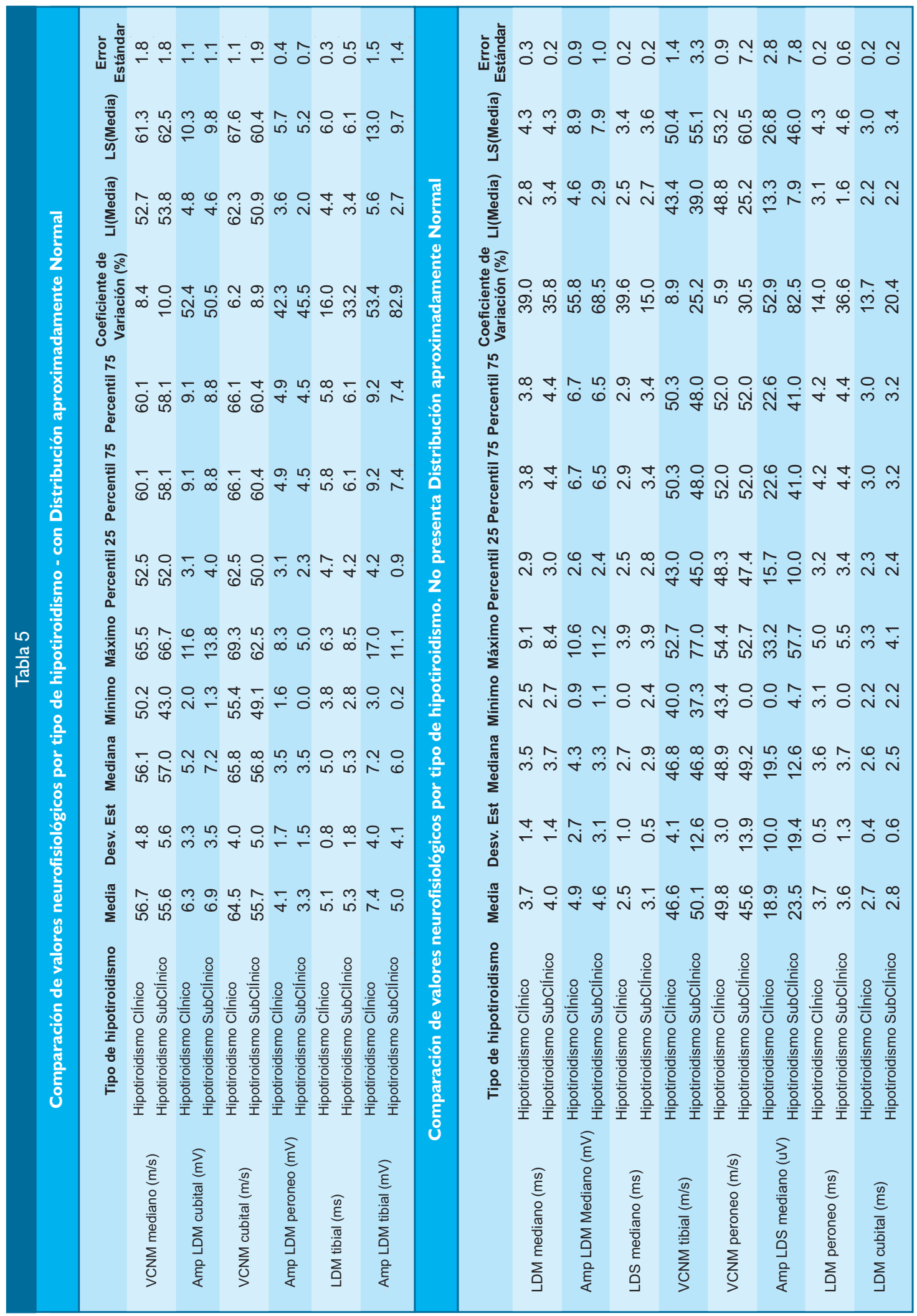


de ANOVA, se observó que la VCNM del Nervio Cubital mostró una diferencia significativa entre los dos tipos de hipotiroidismo, lo cual estaría probablemente directamente relacionado a la duración de la enfermedad, más que al tratamiento específico de la misma, por una posible participación de trastornos metabólicos crónicos que afecten primariamente a las fibras nerviosas sensitivas y luego motoras. En el presente estudio se evidenció una disminución significativa de la velocidad de conducción motora del nervio cubital en los pacientes con HSC en comparación a los pacientes con $\mathrm{HC}$, que difieren de Mai Abdelazeem et al. y Waghmare et al. ${ }^{(23,24)}$ quienes encontraron estudios anormales de conducción nerviosa en los pacientes con hipotiroidismo, predominantemente en los nervios peroneo y mediano; Beghi et al. ${ }^{(25)}$ informaron que los nervios surales seguidos por el mediano sensitivo eran los más predominantes afectados. Esto puede ser debido a diferentes protocolos electrofisiológicos utilizados en los estudios.
Por lo anteriormente descrito y ante un relativo incremento de la prevalencia del hipotiroidismo, por una mayor atención y diagnóstico de esta enfermedad, sería importante considerar como un marcador clínico precoz la presencia de alteraciones sensitivas y/o motoras de los pacientes con sospecha de hipotiroidismo, convirtiendo a las pruebas neurofisiológicas adecuadamente realizadas, en instrumentos muy específicos y de bajo costo para el diagnóstico de éstas.

Agradecimientos: Al Sr. Flavio Romaní Ramos, Licenciado en Estadística, por el análisis estadístico del trabajo de Investigación.

\section{Referencias bibliográficas}

1. Madhuri D, Yasser HO, Burman K. Hypothyroidism. Endocrinol Metab Clin NAm 2007;36:595-615.

2. Hollowell JG, Staehling NW, Flanders WD, et al. Serum TSH, $\mathrm{T} 4$, and thyroid antibodies in the United States population (1988 to 1994): National Health and Nutrition Examination Survey (NHANES III). J Clin Endocrinol Metab 2002;87:489-99.

3. Birte Nygaard. Hypothyroidism (primary). BMJ - Clinical Evidence 2014;02:605.

4. Tunbridge WMG, Evered DC, Hall R, et al. The spectrum of thyroid disease in a community: the Whickham survey. Clin Endocrinol (Oxf) 1977;7:481-93.

5. Canaris GJ, Manowitz NR, Mayor GM, Ridgway EC. The Colorado thyroid disease prevalence study. Arch Intern Med 2000;160:526-34.

6. Weetman AP. Hypothyroidism: screening and subclinical disease. BMJ: British Medical Journal. 1997;314(7088):117578 .

7. Burmeister LA, Ganguli M, Dodge HH, et al. Hypothyroidism and cognition: preliminary evidence for a specific defect in memory. Thyroid 2001;11:1177-1185.

8. García J. et al. Hipotiroidismo subclínico. Actualización. Instituto Nacional de Endocrinología. 2006(12):12:31

9. Misiunas A, Niepomniszcze H, Ravera B, Faraj G, Faure E. Peripheral neuropathy in subclinical hypothyroidism. Thyroid 1995;5:283-86.

10. Ozata M, Ozkardes A, Corakci A, Gandogan MN. Subclinical hypothyroidism does not lead to alteration either in peripheral nerves or in Brain stem Auditory evoked potentials. Thyroid 1995;5:201-5.

11. Monzani F, Caraccio N, et al. Clinical and biochemical features of muscle dysfunction in subclinical hypothyroidism. J Clin Endocrinol Metab. 1997;82(10):3315-18.

12. Kaminsky P, Klein M, Duc M. Hypothyroid Myopathy. physiopathological Approach. Ann Endocrinol. 1992;53(4):125132.

13. Horak HA, Pourmand R. Endocrine myopathies. Neurol Clin. 2000;18(1):203-13.

14. Dyck PJ, Karnes J, O'Brien PC, Swanson CJ. Neuropathy symptom profile in health, motor neuron disease, diabetic neuropathy and amiloidosis. Neurology 1986;36:1300.

15. Nebuchennykh M, Loseth S, Mellgren, SI. Aspects of peripheral nerve involvement in patients with treated hypothyroidism. Eur J Neurol. 2010;17(1):67-72.

16. Beghi E, Delodovici M, Bogliun G, Crespi V and Paleari F et al. Hypothyroidism and polyneuropathy. J Neurol Neurosurg Psychiatry, 1989;52:1420-23.

17. Asia A, Warkar A. Nerve conduction studies in recently diagnosed untreated hypothyroid patients, Indian Journal of Basic and Applied Medical Research, 2015;4(4):330-34.

18. Karne S, Bhalerao N. Nerve conduction studies in patients with primary hypothyroidism. Thyroid Res Pract., 2016;13:131-35.

19. Ajeena I. Prevalence of neuromuscular abnormalities in newly diagnosed patients with thyroid dysfunction. Am J Res Commun., 2013;1:79-88

20. Balaraman A, Natarajan G, Vishwanatha B, Kabali B. A Study of nerve conduction velocity in newly diagnosed hypothyroid females. World Journal of Medical Sciences, 2013;9(4):198-201.

21. Duyff R, Vanden J, Laman D, van Loon B and Linssen W, et al. Neuromuscular findings in thyroid dysfunction: A prospective clinical and electrodiagnostic study. J Neurol Neurosurg Psychiatry, 2000;68:750-55.

22. Cruz M, Tendrich M, Vaisman M, Novis $S$. Electroneuromyography and neuromuscular findings in 16 primary hypothyroidism patients. Arq. Neuropsiquiatr., 1996;54(1):12-18.

23. Mai Abdelazeem et al. Subclinical Peripheral Nerve Affection in Hypothyroidism: The Egyptian Journal of Hospital Medicine Page 2017(67);2:553-63.

24. Waghmare S, Pajai S, Chaudhari R. Motor neuropathy in hypothyroidism: A case-control study. The Health Agenda, 2015;3(3):1.

25. Beghi E, Delodovici M, Bogliun G, Crespi V, Paleari F, et al. Hypothyroidism and polyneuropathy. J Neurol Neurosurg Psychiatry, 1989;52:1420-23.

Citar como: Pérez-Galdos P. Paucar-Castillo R. Análisis clínico y electrofisiológico del hipotiroidismo subclínico en una población hospitalaria. Diagnóstico(Lima).2019;58(2):61-6. DOI: https://doi.org/10.33734/diagnostico.v58i2.204

Correspondencia: Proel Pérez-Galdos Dirección: Jirón JoséAntonio Roca 150 Dpto 1604. Santa Beatriz-Cercado de Lima. Teléfono: 998706946

Correo electrónico: pproel@yahoo.com 